\title{
Tecnologías de Información y Comunicación como herramientas de apoyo en los procesos académicos en instituciones de educación superior
}

\section{Information and Communication Technologies as support tools in the academic processes in higher education institutions}

\section{Las TIC como herramienta de apoyo en los procesos académicos}

\author{
Rossana Narcisa Guillén Sánchez ${ }^{(1)}$ \\ Matilde Flores-Urbáez ${ }^{(2)}$ \\ Gregorio Palma Macías ${ }^{(3)}$ \\ Carlos Rivero Torres ${ }^{(4)}$ \\ (1) Universidad Técnica de Manabí, Ecuador. \\ (2) Universidad Técnica de Manabí, Ecuador. email: floresurbaez8@gmail.com \\ (3) Universidad Técnica de Manabí, Ecuador. email: gpalma@utm.edu.ec \\ (4) Universidad Técnica de Manabí, Ecuador. email: crivero@utm.edu.ec
}

Aprobado: 08-06-2020

\author{
Contacto: \\ floresurbaez8@gmail.com
}

\section{Resumen}

El objetivo de esta investigación es analizar la percepción que tienen los docentes de la Facultad de Ciencias Administrativas y Económicas de la Universidad Técnica de Manabí sobre el uso de Tecnologías de Información y Comunicación para el desarrollo de sus labores académicas. Esta investigación fue de campo y su diseño no experimental transversal. La información se recolectó a través de un cuestionario en escala de Likert, la confiabilidad de este instrumento se hizo a través del coeficiente Alfa de Cronbach, los resultados de las encuestas se procesaron con el programa Excel año 2016 y se presentaron en tablas de frecuencia. La población de la investigación fue de 98 docentes con nombramiento adscritos a la Facultad de Ciencias Administrativas y Económicas de la Universidad Técnica de Manabí. La muestra fue de tipo no probabilística o dirigida, con un total de 20 individuos. Los resultados indican que para los docentes que participaron en el estudio el uso de Tecnologías de Información y Comunicación son de suma importancia para la administración de la docencia y no solo para impartir clases presenciales o virtuales. Sin embargo, consideran que deben mejorarse los procesos de capacitación en el uso de estas tecnologías. Se concluye que los docentes de la Facultad de Ciencias Administrativas y Económicas reconocen que no pueden separar las tecnologías de información, comunicación del desempeño de sus funciones, pero es clave una adecuada capacitación para su uso y máximo aprovechamiento.

Palabras clave: gestión de tecnologías de información y comunicación, gestión de procesos académicos, funciones sustantivas, instituciones de educación superior

\begin{abstract}
The objective of this investigation is to analyze the perception that the professors of the Faculty of Administrative and Economic Sciences of the Technical University of Manabí have about the use of Information and Communication Technologies for the development of their academic work. This research was in the field and its non-experimental cross-sectional design. The information was collected through a Likert scale questionnaire, the reliability of this instrument was made through the Cronbach's Alpha coefficient, the results of the surveys were processed with the Excel 2016 program and were presented in frequency tables. The research population consisted of 98 appointed teachers assigned to the Faculty of Administrative
\end{abstract}


and Economic Sciences of the Technical University of Manabí. The sample was non-probabilistic or directed, with a total of 20 individuals. The results indicate that for the teachers who participated in the study, the use of Information and Communication Technologies is of utmost importance for the administration of teaching and not only for teaching face-to-face or virtual classes. However, they consider that the training processes in the use of these technologies should be improved. It is concluded that the teachers of the Faculty of Administrative and Economic Sciences recognize that they cannot separate information and communication technologies from the performance of their duties, but adequate training for their use and maximum use is key.

Key words: management of information and communication technologies, management of academic processes, substantive functions, higher education institutions.

\section{Introducción}

En este nuevo milenio las universidades se enfrentan a nuevos retos para brindar una formación de calidad e incorporar a sus entornos de aprendizajes distintas herramientas tecnológicas. Esto conlleva a que los docentes tienen el compromiso de integrar las Tecnologías de Información y Comunicación en el proceso enseñanza-aprendizaje, así como de cambiar sus métodos de enseñanza apoyados por tecnologías que permitan a los estudiantes trabajar de una manera colaborativa, cooperativa y en forma sincrónica o asincrónica (San Andrés, Pazmiño, Mero y Pinargote 2018).

En los últimos años la Universidad Técnica de Manabí se ha visto fortalecida con la implementación de banda ancha que ha hecho accesible la Internet y herramientas informáticas de gestión de las funciones académicas y administrativas a toda la comunidad universitaria. Esto se logró con la creación de una plataforma institucional a través de la cual se puede obtener información institucional relevante y pertinente sobre todos los procesos sustantivos (docencia, investigación y vinculación con la sociedad) y de los administrativos.

Sin embargo, aun cuando es evidente los beneficios que proporciona la automatización de los procesos institucionales en la Universidad, es importante conocer qué piensan los docentes de la Universidad Técnica de Manabí, como usuarios, del hecho de que deben realizar la planificación, la organización y el control de sus actividades académicas desde una plataforma informática.

La presente investigación tiene como objetivo, analizar la percepción que tienen los docentes de la Facultad de Ciencias Administrativas y Económicas de la Universidad Técnica de Manabí sobre el uso de Tecnologías de Información y Comunicación para el desarrollo de sus labores académicas.

\section{Algunas generalidades sobre el uso de las tecnologías de información y comunicación en instituciones de educación superior}

Es indiscutible que dentro de las diversas herramientas de enseñanza y aprendizaje se encuentren las tecnologías de la información y comunicación, las cuales se constituyen en metodologías activas que el docente puede utilizar para motivar a un mejor aprendizaje, con el propósito de desarrollar de manera más rápida y eficiente los procesos educativos (Moreira y Cedeño, 2017).

Las Tecnologías de Información y Comunicación (TIC) han evolucionado y modificado aceleradamente el funcionamiento de las organizaciones, la inclusión de estas tecnologías en la educación está haciendo cambiar de forma reveladora la relación de los docentes con los estudiantes, desde la forma de impartir las clases hasta los procesos de evaluación. Cada vez aparecen nuevos recursos de TIC por lo que se establece como una obligación la capacitación y la adaptabilidad a las nuevas posibilidades que éstas brindan. Esto crea nuevos desafíos en el campo de la educación superior que permiten renovar de manera gradual los procesos didácticos por el ilimitado acceso a recursos de información científica y las formas de comunicación entre docentes y estudiantes, así como los procesos de enseñanza y aprendizaje que se promueven por la Internet (Cedeño y Vélez 2017).

La tendencia de la educación superior es la implementación de las TIC en la docencia, debido a las facilidades que hoy en día tienen los estudiantes para acceder a ellas para educarse e informarse, en un mundo donde las herramientas tecnológicas y los medios digitales ocupan un lugar significativo (Sánchez, Pardo, Izquierdo (2010, citado por Novillo, Espinosa y Guerrero, 2017).

Reinoso y Tintin (2012, citado por Rodríguez 2016) comentan:

En el Ecuador, según el Plan Nacional del Buen Vivir 2009-2013 se estableció en la Política 2.7 la 
promoción del acceso a la información y a las tecnologías de la información y comunicación para incorporar a la población a la sociedad de la información y fortalecer el ejercicio de la ciudadanía. Por medio de esta política de Estado se espera facilitar y democratizar el acceso a las TIC con el objetivo de promover el desarrollo de capacidades para el uso de plataformas, bancos de información, educación, entre otros (p. 2).

Estudios realizados sobre el estado de las TIC en América Latina indican que existe una brecha digital y económica entre América Latina y los países desarrollados, señalando el bajo desempeño de América Latina en la infraestructura y uso de las TIC, que puede estar afectando la productividad, y otros factores como la innovación, la educación y el crecimiento económico Quiroga y Murcia (2015, citado por Salto-Rivas, Novoa-Hernández y SerranoRodríguez, 2019).

De acuerdo al Portal Web Internet World Stats (2018), las estadísticas en 2012 con respecto al uso del computador señalan que Ecuador ocupó el lugar 65 y en 2016 se situó en el puesto 71 en cuanto al uso de Internet. A escala nacional, la información recopilada por el INEC (2016) el 36,0\% de los hogares tiene acceso a Internet, de ellos el $24,5 \%$ accede a través de algún medio inalámbrico, 4,0 puntos más que en 2012. En cuanto al uso de computadora por provincias, Galápagos es la provincia con mayor número de personas que utiliza computadora con el $68,4 \%$, seguida por Pichincha con el $65,2 \%$; mientras que la provincia que registra menor uso es Los Ríos con el 35,4\%; y, Manabí $41.5 \%$. A nivel nacional, el $38,0 \%$ de las personas usó Internet como fuente de información, mientras el $31,5 \%$ lo utilizó como medio de comunicación en general.

La Constitución de la República de Ecuador (2008), en el Art. 347 numeral 8, establece que será compromiso del Estado "Incorporar las tecnologías de la información y comunicación en el proceso educativo y propiciar el enlace de la enseñanza con las actividades productivas y sociales". Así también la Ley Orgánica de Educación Intercultural (2011), en su Art. 6 respalda el uso de las TIC en los procesos educativos para el desarrollo de actividades productivas o sociales. Por otro lado, la Ley Orgánica de Educación Intercultural (2017), hace énfasis en que "El desarrollo profesional es un proceso permanente e integral de actualización psicopedagógica y en ciencias de la educación" (Art. 112).
Hoyos (2013, citado por Rivera y Suconota 2018) indica:

En el año 2008, la UNESCO propuso "Estándares sobre competencia en TIC para docentes" como referencia mundial en cuanto a los conocimientos, habilidades y destrezas que debe poseer el docente y que impliquen el dominio de las competencias digitales para hacer firme la incorporación de las TIC dentro y fuera de las aulas (p. 3).

Las instituciones de educación superior de acuerdo con la Ley Orgánica de Educación Superior del Ecuador y su Reglamento, hacen énfasis en la aplicación de TIC debe estar acorde con el desarrollo científico tecnológico de la sociedad del siglo XXI.

La Universidad Técnica de Manabí durante los últimos años se ha visto fortalecida con la implementación de banda ancha para la accesibilidad a la Internet de toda la comunidad universitaria, por ello a través de su página web se puede obtener información relacionada con la gestión de las distintas funciones sustantivas.

\section{Materiales y métodos}

El estudio se realizó en la Facultad de Ciencias Administrativas y Económicas de la Universidad Técnica de Manabí (UTM), ubicada en el cantón Portoviejo, provincia de Manabí, en Ecuador. La Facultad de Ciencias Administrativas y Económicas fue creada el 25 de noviembre de 1977, mediante Resolución $\mathrm{N}^{\circ} 4043$ expedida por el Honorable Consejo Universitario de la UTM con dos carreras: Administración de Empresas y Economía. Posteriormente, el 28 de abril de 1989 se crea la carrera de Contabilidad y Auditoría y el 16 de agosto 2019 la carrera de Turismo.

Para la matrícula del período académico octubre 2019 - febrero 2020, en la UTM se matricularon 1213 estudiantes en la carrera de Administración de Empresas, 229 en Economía en la modalidad en línea y 1274 estudiantes en la modalidad presencial, 1440 en la carrera de Contabilidad y Auditoría y 165 en la Carrera de Turismo en la modalidad en línea y 56 en la modalidad presencial (Secretaria de la Facultad de Ciencias Administrativas y Económicas/UTM, 2020). Su planta docente actualmente está conformada por 98 docentes con nombramiento y 23 docentes por contrato, 40 docentes con nombramiento se encuentran realizando sus estudios doctorales y 17 docentes han obtenido su grado académico de Doctor (Coordinación de las Carreras 
de la Facultad de Ciencias Administrativas y Económicas/UTM, 2020).

Esta investigación fue de campo, el diseño no experimental transversal porque se recabó la información en un momento único, y el alcance fue exploratorio - descriptivo (Hernández Sampieri y Mendoza-Torres, 2018). La información se recolectó a través de un cuestionario en escala de Likert con 6 items al cual se le calculó la confiabilidad a través del coeficiente Alfa de Cronbach con un valor de 0,86 . lo que indica que los resultados obtenidos de la aplicación del cuestionario a los docentes de la Facultad de Ciencias Administrativas y Económicas de la UTM son consistentes y coherentes. Una vez determinada la confiabilidad el cuestionario, se procedió a entregarlos en impreso a los docentes para ser contestados. Los resultados de las encuestas se procesaron con el programa Excel año 2016 y se presentaron en tablas de frecuencia.

La población de la investigación estuvo conformada por los 98 docentes con nombramiento adscritos a la
Facultad de Ciencias Administrativas y Económicas de la UTM. La muestra fue de tipo no probabilística o dirigida (Hernández Sampieri y Mendoza-Torres, 2018), tomando como criterio aquellos docentes que voluntariamente accedieran a participar en el estudio, lo cual permitió garantizar la franqueza de las respuestas. La muestra tuvo un total de 20 docentes.

\section{Resultados}

Los resultados se presentan, en las tablas de frecuencia, se aprecian las respuestas de los docentes de la Facultad de Ciencias Administrativas y Económicas de la UTM. Los resultados evidencian que el $100 \%$ de los encuestados están Totalmente de acuerdo en que el uso de TIC en clave en el ámbito educativo.

\begin{tabular}{|l|l|l|}
\hline Alternativas de respuesta & Frecuencia & Porcentaje \\
\hline Totalmente de acuerdo & 20 & 100 \\
\hline De acuerdo & 0 & 0 \\
\hline Ni de acuerdo ni en desacuerdo & 0 & 0 \\
\hline En desacuerdo & 0 & 0 \\
\hline Totalmente en desacuerdo & 0 & 0 \\
\hline TOTAL & 20 & 100 \\
\hline
\end{tabular}

Tabla 1. El uso de TIC es clave en el ámbito educativo

En lo concerniente al uso de las TIC, y su importante en el aula de clases, en la tabla 2 se puede observar que un porcentaje importante de la muestra $(85 \%)$

\begin{tabular}{|l|l|l|}
\hline Alternativas de respuesta & Frecuencia & Porcentaje \\
\hline Totalmente de acuerdo & 17 & 85 \\
\hline De acuerdo & 2 & 10 \\
\hline Ni de acuerdo ni en desacuerdo & 1 & 5 \\
\hline En desacuerdo & 0 & 0 \\
\hline Totalmente en desacuerdo & 0 & 0 \\
\hline TOTAL & 20 & 100 \\
\hline
\end{tabular}

Tabla 2.El uso de las TIC es importante en el aula de clases.

En la tabla 3 se evidencia que el $90 \%$ de los docentes que participaron en el estudio están totalmente de está totalmente de acuerdo en que es importante usar TIC en el aula de clases.

\begin{tabular}{|l|l|l|}
\hline Alternativas de respuesta & Frecuencia & Porcentaje \\
\hline Totalmente de acuerdo & 18 & 90 \\
\hline De acuerdo & 2 & 10 \\
\hline Ni de acuerdo ni en desacuerdo & 0 & 0 \\
\hline En desacuerdo & 0 & 0 \\
\hline Totalmente en desacuerdo & 0 & 0 \\
\hline TOTAL & 20 & 100 \\
\hline
\end{tabular}


Tabla 3. El uso de las TIC optimiza el proceso de administración de la docencia

En cuanto al tema de las aulas virtuales y su utilización para fortalecer la docencia, en la tabla 4, se evidencia que el $85 \%$ de los docentes encuestados

\begin{tabular}{|l|l|l|}
\hline Alternativas & Frecuencia & Porcentaje \\
\hline Totalmente de acuerdo & 17 & $85 \%$ \\
\hline De acuerdo & 3 & $15 \%$ \\
\hline Ni de acuerdo ni en desacuerdo & 0 & $0 \%$ \\
\hline En desacuerdo & 0 & $0 \%$ \\
\hline Totalmente en desacuerdo & 0 & $0 \%$ \\
\hline Total & 20 & $100 \%$ \\
\hline
\end{tabular}

Tabla 4: El uso de aulas virtuales favorece la función de docencia están totalmente de acuerdo en que el uso de aulas virtuales favorece la docencia.

Los resultados reflejan en la tabla 5, evidencian que el $85 \%$ de los docentes encuestados están totalmente plataforma de la UTM en los procesos de acuerdo en que es importante el uso de la administrativos.

\begin{tabular}{|l|l|l|}
\hline Alternativas & Frecuencia & Porcentaje \\
\hline Totalmente de acuerdo & 17 & 85 \\
\hline De acuerdo & 3 & 15 \\
\hline Ni de acuerdo ni en desacuerdo & 0 & 0 \\
\hline En desacuerdo & 0 & 0 \\
\hline Totalmente en desacuerdo & 0 & 0 \\
\hline Total & 20 & 100 \\
\hline
\end{tabular}

Tabla 5. La plataforma de la UTM es una herramienta de utilidad para el manejo de los procesos administrativos

En el análisis de los resultados en la tabla 6 no revelan homogeneidad en las respuestas como en los cuadros anteriores. Por el contrario, evidencia diversidad de opiniones en el nivel de satisfacción de los participantes en cuanto a las capacitaciones recibidas en el uso de TIC. Como se observa, la mayoría de los docentes que participaron en el estudio (70 por ciento) se ubican en las categorías de respuesta de connotación positiva al considerar estar totalmente de acuerdo y de acuerdo en que las capacitaciones recibidas en la UTM en el uso de TIC son las más apropiadas. Otro porcentaje importante ( 25 por ciento) se encuentra en un nivel intermedio en cuanto a considerar apropiadas las capacitaciones recibidas en la UTM en el uso de TIC. Solo un 5 por ciento manifestó estar en desacuerdo.
Los resultados de las encuestas que se aplicaron a los docentes de la Facultad de Ciencias Administrativas y Económicas de la Universidad Técnica de Manabí, indican que para los encuestados el uso de TIC para la docencia y para la administración de la docencia es sumamente importante, no solo para impartir clases presenciales, sino que también valoran su uso para la educación virtual. En esta investigación se concuerdan con los siguientes autores (San Andrés, Pazmiño, Mero, y Pinargote), destacan el compromiso de los docentes de integrar las TIC en el proceso enseñanza-aprendizaje, así como de cambiar sus métodos de enseñanza apoyados por tecnologías que permitan a los estudiantes trabajar de una manera colaborativa, cooperativa y en forma sincrónica o asincrónica.

\section{Discusión}

\begin{tabular}{|l|l|l|}
\hline Alternativas de respuesta & Frecuencia & Porcentaje \\
\hline Totalmente de acuerdo & 4 & $20 \%$ \\
\hline De acuerdo & 10 & $50 \%$ \\
\hline Ni de acuerdo ni en desacuerdo & 5 & $25 \%$ \\
\hline En desacuerdo & 1 & $5 \%$ \\
\hline Totalmente en desacuerdo & 0 & $0 \%$ \\
\hline Total & 20 & $100 \%$ \\
\hline
\end{tabular}

Tabla 6. Las capacitaciones recibidas en la UTM en el uso de TIC son las más apropiadas 
Aunque la mayoría de los encuestados consideraron que las capacitaciones recibidas en la UTM en el uso de TIC son las más apropiadas, un porcentaje importante manifestó estar ni de acuerdo ni en desacuerdo y en desacuerdo con esta opinión. Estos resultados en cierta forma coinciden con los del estudio de Moreira y Cedeño (2017) cuando analizaron las tecnologías de la información y su incidencia en la formación de profesionales en la Carrera de Bibliotecología y Ciencias de la Información de la UTM. Los resultados de este estudio demostraron que en esa carrera se presentan deficiencias en la capacitación docente para hacer uso de las tecnologías de la información dentro del desarrollo de sus actividades académicas. Habría que analizar las causas de la insatisfacción en las capacitaciones en TIC expresadas por los docentes de la Facultad de Ciencias Administrativas y Económicas de la UTM para implementar los correctivos apropiados y mejorar los planes de capacitación en el futuro.

No considerar apropiadas las capacitaciones en el uso de TIC pudiera traer como consecuencia lo que se evidenció en el estudio de Ponce y Moran (2017), en el cual se determinó el grado de conocimiento y utilización de TIC y de herramientas web 2.0 que los docentes de la Universidad Técnica de Manabí emplean en la mediación pedagógica. Los resultados indicaron que los docentes que participaron en el estudio aprovechaban parcialmente las tecnologías en el proceso formativo, porque no tenían verdaderas competencias digitales. Puede considerarse que esto también está asociado con la calidad y cantidad de las capacitaciones que ofrece la Institución a sus docentes.

\section{Conclusiones}

Se puede concluir que, en general, la percepción que tienen los docentes en cuanto a la importancia del uso de TIC en los procesos de enseñanza aprendizaje y en gestión administrativa es muy positiva, lo que es un importante punto a favor para que la UTM aproveche esa apertura de sus docentes y les proporcione las herramientas tecnológicas apropiadas y se puedan implementar en el desarrollo de las tres funciones sustantivas. No obstante, se requiere que la universidad revise los mecanismos de capacitación en TIC y más aún en este momento en el cual dada la situación de la pandemia por el COVIT- 19, se ha tenido que migrar completamente al uso de TIC para desempeñar la además de la gestión administrativa de toda la Universidad.
Esto implica que el docente de la Facultad de Ciencias Administrativas y Económicas reconoce que no puede separar las TIC del desempeño de sus funciones, pero es clave una adecuada capacitación para su uso y máximo aprovechamiento.

\section{Bibliografía}

Asamblea Nacional del Ecuador. (2011). Ley Orgánica de Educación Intercultural. Marco Legal Educativo. Recuperado de: http://www.academia.edu/download/549 73683/Marco_Legal_Educativo_2012.pd f

Cedeño, S. y Vélez, J. (2017). La formación profesional mediante seminarios web: Guía para docentes y estudiantes. Revista Atlante: Cuadernos de Educación y Desarrollo. Recuperado de: http://www.eumed.net/rev/atlante/2017/0 7/seminarios-web-formacion.htm, http://hdl.handle.net/20.500.11763/atlant e1707seminarios-web-formacion

Hernández-Sampieri, R. y Mendoza-Torres, C. (2018). Metodología de la Investigación. Editorial Mc Graw-Hill: México. Instituto Nacional de Estadísticas y Censo/INEC (2016). Ecuador en cifras. Recuperado de: https://www.ecuadorencifras.gob.ec/docu mentos/webnec/EstadisticasSociales/iTIC /2016/170125.Presentacion_Tics_2016.p df

San Andrés, E.; Pazmiño, M.; Mero, K. y Pinargote, C. (2018). Las herramientas de la web 2.0 en la mediación pedagógica universitaria. Revista Universidad Ciencia y Tecnología. Nro. Especial 03, 111-121. Recuperado de:

https://www.uctunexpo.autanabooks.com index.php/uct/article/view/94

Moreira, E. y Cedeño, K. (2017). Las Tecnologías de la información y su incidencia en la formación de profesionales en las carreras de Bibliotecología y Ciencias de la Información de la Universidad Técnica De Manabí. Revista Atlante. Cuadernos de Educación y Desarrollo. Recuperado de:

http://www.eumed.net/rev/atlante/2017/0 8/bibliotecologia-manabi.html 
Novillo, E., Espinosa, M. y Guerrero, J. (2017). Influencia de las TIC en la Educación Universitaria, caso Universidad Técnica de Machala. Revista: INNOVA Research Journal 2, (3), 69-79. Recuperado de: https://revistas.uide.edu.ec/index.php/inn ova/article/view/136/972

Ponce y Moran (2017). Las TIC como medio de aprendizaje de los idiomas. Revista Cognosis. 2(3). DOI: https://doi.org/10.33936/cognosis.v2i3.85 $\underline{7}$

Universidad Técnica de Manabí. (2014). Reglamento Orgánico de Gestión Organizacional por Procesos. Recuperado de: https://www.utm.edu.ec/launiversidad/reglamentos/file/575reglamento-organico-de-gestionorganizacional-por-procesos-de-la-utm2014? start $=20$

Universidad Técnica de Manabí (2020). Matriculados período académico octubre 2019 - febrero 2020. Secretaria de la Facultad de Ciencias Administrativas y Económicas.

Universidad Técnica de Manabí (2020). Reporte de docentes por categoría (nombramiento, contrato). Coordinación de Carreras de la Facultad de Ciencias Administrativas y Económicas.

Rivera, D. \& Suconota, E. (2018). Las TIC en la gestión de los procesos educativos. Razón y palabra. Primera Revista Electrónica en
Iberoamérica Especializada en Comunicación.22, (3)_102. 481-509. Recuperado de: http://revistas.comunicacionudlh.edu.ec/i ndex.php/ryp

Rodríguez, E. (2016). Análisis de la competitividad de las Pymes de desarrollo de Software del Distrito Metropolitano de Quito asociadas a la AESOFT durante el período 2014-2015. Tesis Maestría en Gerencia Empresarial (FCA).163 hojas. Quito. Repositorio Digital Escuela Politécnica Nacional. Recuperado de: http://bibdigital.epn.edu.ec/handle/15000 $\underline{17036}$

Saltos-Rivas, R.; Novoa-Hernández, P. y Serrano Rodríguez, R. (2019). Evaluación de la presencia de competencias digitales en las Instituciones de Educación Superior en América Latina. Revista Ibérica de Sistemas e Tecnologias de Informação. 23-36. Recuperado de: https://www.researchgate.net/profile/Roc io_Serrano3/publication/336239735_Eva luation_of thepresence_of_digital_comp etences_in_higher_education_institutions _Evaluacion_de_la_presenciade_compet encias_digitales_en_las_Instituciones_de _Educacion_Superior_en_America_Latin a/links/5d960a62a6fdccfd0e743163/Eval uation-of-the-presence-of-digitalcompetences-in-higher-educationinstitutions-Evaluacion-de-la-presenciade-competencias-digitales-en-lasInstituciones-de-Educacion-Superior-enAmerica-Latina.pdf 